Case Report

\title{
Nonfatal Hyperammonemic Encephalopathy as a Late Complication of Roux-en-Y Gastric Bypass
}

\author{
Juan D. Salcedo (iD, ${ }^{1}$ Jordan S. Goldstein, ${ }^{1}$ \\ Jose M. Quinonez, ${ }^{2}$ and Maria Antonietta Mosetti $^{3}$ \\ ${ }^{1}$ Department of Internal Medicine, University of Miami Miller School of Medicine, Miami, FL, USA \\ ${ }^{2}$ University of Miami Miller School of Medicine, Miami, FL, USA \\ ${ }^{3}$ Division of Hospital Medicine, University of Miami Medical Towers, University of Miami Miller School of Medicine, Miami, FL, USA
}

Correspondence should be addressed to Juan D. Salcedo; juan.salcedobetanco@jhsmiami.org

Received 24 March 2019; Accepted 11 June 2019; Published 1 July 2019

Academic Editor: Yoshihiro Moriwaki

Copyright (C) 2019 Juan D. Salcedo et al. This is an open access article distributed under the Creative Commons Attribution License, which permits unrestricted use, distribution, and reproduction in any medium, provided the original work is properly cited.

\begin{abstract}
Roux-en-Y gastric bypass (RYGB) is the most common weight loss procedure performed in the US. Gastric bypass-related hyperammonemia (GaBHA) is a potentially fatal entity, characterized by encephalopathy associated with hyperammonemia and various nutritional deficiencies, which can present at variable time intervals after RYGB. Twenty-five cases of hyperammonemic encephalopathy after bariatric surgery have been previously reported in the literature. We describe the case of a 48 -year-old Hispanic woman with no prior history of liver disease, presenting with nonfatal hyperammonemic encephalopathy as a late postoperative complication 20 years after undergoing a RYGB. Hyperammonemic encephalopathy in the absence of known hepatic dysfunction presents a diagnostic dilemma. An early diagnosis and intervention are crucial to decrease morbidity and mortality.
\end{abstract}

\section{Introduction}

Roux-en-Y gastric bypass (RYGB) is the most common weight loss procedure performed in the US $[1,2]$. It achieves successful and sustained weight loss in morbidly obese patients, through restrictive and malabsorptive mechanisms [3].

Although RYGB has lower morbidity and mortality rates compared to other bariatric procedures, there are numerous postoperative complications which may occur [4]. Early postoperative complications include gastrointestinal leaks, venous thromboembolic disease, pulmonary and cardiovascular complications, and tracheal reintubation [5]. The average 30-day mortality rate reported for RYGB is $0.2 \%$ [6]. Late postoperative complications include anastomotic stricture, bowel obstruction, fistula formation, intussusception, marginal ulcers, gallstone formation, dumping syndrome, hypoglycemia, malnutrition, fat malabsorption, and vitamin deficiencies [7].

Neurologic complications after bariatric surgery have a reported prevalence of 5\% [8], including optic neuropathy, polyradiculoneuropathy, myelopathy, and encephalopathy. Of these, encephalopathy is a rare complication, usually occurring early, and generally attributed to thiamine deficiency [9]. Gastric bypass-related hyperammonemia (GaBHA) has been recently recognized as a life-threatening distinct entity, characterized by encephalopathy associated with hyperammonemia, elevated plasma glutamine, hypoalbuminemia, reactive hypoglycemia, low zinc, and other nutritional deficiencies. This condition has a high fatality rate reported around 50\% and has a predilection for middleaged women without prior liver disease who have achieved successful weight loss after RYGB [10]. It can occur at variable time intervals after RYGB, ranging from 1 month to 28 years after the surgery [11]. Hyperammonemic encephalopathy in the absence of known hepatic dysfunction presents a diagnostic dilemma. Therefore, an early diagnosis and intervention are crucial to prevent a rapid decline that may occur when the diagnosis is unrecognized.

\section{Methods}

We conducted a MEDLINE database query by combining the search terms "Hyperammonemia", "Encephalopathy", and "Bariatric surgery". The resultant seven articles 
[10-16] were pulled from the literature. English language article titles and abstracts were screened and the appropriate articles reviewed. As far as we could determine in our search, twenty-five cases of hyperammonemic encephalopathy after bariatric surgery have been previously reported in the literature.

\section{Case Presentation}

A 48-year-old Hispanic female with a past medical history of multiple bariatric surgical procedures, presented with increasing lower extremity edema secondary to worsening chronic lymphedema.

During her hospitalization she developed altered mental status in the setting of elevated serum ammonia levels, despite having no history of liver disease.

Vertical banded gastroplasty was initially performed at age 28 , but it failed to produce adequate weight loss. Consequently, three years later, she underwent open surgical revision which was converted to an open RYGB with concomitant cholecystectomy. She had lost over $100 \mathrm{~kg}$ since the first bariatric procedure (166 kg preoperatively to $63 \mathrm{~kg}$ on presentation). However, she developed multiple complications, including chronic lower extremity lymphedema, small bowel obstruction requiring multiple surgical interventions, malabsorption secondary to short bowel syndrome, multiple vitamin deficiencies, wet beriberi requiring chronic thiamine replacement, osteoporosis causing a left hip fracture, and chronic low back pain.

In July 2018, she initially presented to the emergency department with increasing lower extremity edema secondary to chronic lymphedema. On arrival, her vital signs were within normal limits. Her physical exam was remarkable for bilateral lower extremity pitting edema extending to the hips and an otherwise unremarkable exam. A chest $\mathrm{x}$-ray disclosed no cardiomegaly, pulmonary congestion or pleural effusion. Her last echocardiogram showed an ejection fraction of $60 \%$ with no wall motion abnormalities.

During the admission, the patient received intravenous bumetanide with significant improvement. However, she developed waxing and waning episodes of confusion, and on day 3 of her hospitalization became overtly encephalopathic. She was disoriented to person, time, and space, with bizarre affect as well as incoherent speech, unable to recognize her close relatives. On physical exam patient was lethargic and had asterixis. According to her family members, the patient had previous episodes where she would become lethargic, but never to this degree.

Laboratory results were significant for an elevated plasma ammonia level of $173 \mu \mathrm{mol} / \mathrm{L}$ (normal range 11 - $50 \mu \mathrm{mol} / \mathrm{L}$ ), despite downtrending transaminases (aspartate transaminase of $32 \mathrm{U} / \mathrm{L}$ and alanine transaminase of $55 \mathrm{U} / \mathrm{L})$. Results were also significant for macrocytic anemia (hemoglobin $10.3 \mathrm{~g} / \mathrm{dL}$ and mean corpuscular volume 107/5), a low serum albumin of $2.3 \mathrm{~g} / \mathrm{dL}$, low serum protein of $4.1 \mathrm{~g} / \mathrm{dL}$, alkaline phosphatase of $122 \mathrm{U} / \mathrm{L}$, total bilirubin of $0.5 \mathrm{mg} / \mathrm{dL}$, and an elevated international normalized ratio (INR) of 1.65. Serum glucose and zinc levels were borderline low at 62 and 54 (normal range $56-134 \mu \mathrm{g} / \mathrm{dL}$ ), respectively. The laboratory findings are further outlined in Table 1. Additionally, her plasma salicylate level, acetaminophen level, and urine toxicology were all negative. Despite a history of vitamin deficiencies, she was receiving chronic thiamine and vitamin B12 replacement at home, with normal values on admission. Noncontrast computed tomography (CT) imaging of the head disclosed no evidence of acute infarction, intracranial hemorrhage, or mass effect. The patient had no history of prior liver conditions or cirrhosis, and even though she presented elevation of transaminases and the INR, these abnormalities resolved once her condition improved. The patient had no other laboratory findings for compatible signs of cirrhosis (no thrombocytopenia or hyperbilirubinemia), neither physical stigmata suggestive of acute or chronic liver disease. Abdominal ultrasound was performed to further elucidate liver panel abnormalities and demonstrated only mild hepatic steatosis with no evidence of cirrhosis or ascites.

She was treated with supportive care and lactulose. The following day she had numerous bowel movements, and her serum ammonia level came down to $24 \mu \mathrm{mol} / \mathrm{L}$. Given the fast clinical improvement and resolution of laboratory abnormalities after supportive treatment, a liver biopsy was not indicated. Once her clinical condition improved and her mental status returned to normal, the patient was discharged from the hospital.

\section{Discussion}

Ammonia is extremely toxic to the central nervous system [17]. It is a bi-product of protein catabolism from amino acid deamination (whether from a high-protein diet, prolonged starvation, or naturally produced by gut flora) [18]. The urea cycle catabolizes toxic ammonia into urea, a process that occurs predominantly in the liver. Urea is nontoxic and is ultimately excreted by the kidneys [18].

In the urea cycle (Figure 1), ammonia is the source of the first amino group, required in the synthesis of carbamoyl phosphate, which happens in the hepatocyte mitochondria. The second amino group comes from glutamate in the formation of aspartate, which happens in the hepatocyte cytoplasm. This reaction produces $\alpha$-ketoglutarate, which then becomes available for the Krebs cycle [18]. In the muscle and peripheral tissues (including the brain astrocytes), glutamate accepts free ammonia produced by amino acid catabolism. Glutamine transports ammonia from the peripheral tissues to the liver, where glutamine is broken back to glutamate and ammonia via glutaminase $[19,20]$. An increase in free blood ammonia has shown to accumulate glutamine within the astrocytes, which has osmotic effects and causes astrocyte swelling and cerebral edema [21]. The normal rate of urea cycle far exceeds the ammonia production rate by protein catabolism. Therefore, slight elevations in the serum free ammonia concentration $(>60 \mu \mathrm{mol} / \mathrm{L})$ reflect an impairment in the urea cycle, independent of the etiology [19]. This impairment may cause a wide range of neurologic manifestations including asterixis, changes in behavior, slurred speech, 
TABLE 1: Laboratory findings during admission.

\begin{tabular}{|c|c|c|c|c|}
\hline Laboratory & Day of Admission & Day 3 & Day 4 (discharge) & Normal range \\
\hline Ammonia $(\mu \mathrm{mol} / \mathrm{L})$ & NA & 173 & 24 & $11-50 \mu \mathrm{mol} / \mathrm{L}$ \\
\hline Hemoglobin $(\mathrm{g} / \mathrm{dL})$ & 10.3 & 10.5 & 10.5 & $11.1-14.6 \mathrm{~g} / \mathrm{dL}$ \\
\hline Mean Corpuscular Volume & 107.5 & 106.5 & 107.9 & 79.9 - 95.0 fL \\
\hline Platelets $(103 / \mathrm{uL})$ & 152 & 179 & 341 & $140-400103 / \mathrm{uL}$ \\
\hline White Blood Cells (103/uL) & 7.0 & 7.5 & 5.3 & $4.0-10.5103 / \mathrm{uL}$ \\
\hline Glucose $(\mathrm{mg} / \mathrm{dl})$ & 81 & 62 & 85 & $65-99 \mathrm{mg} / \mathrm{dL}$ \\
\hline $\operatorname{Zinc}(\mu \mathrm{g} / \mathrm{dL})$ & 54 & NA & NA & $56-134 \mu \mathrm{g} / \mathrm{dL}$ \\
\hline Vitamin B1 (nmol/L) & 191.9 & $\mathrm{ND}$ & $\mathrm{ND}$ & $66.5-200.5(\mathrm{nmol} / \mathrm{L})$ \\
\hline Vitamin B12 (pg/mL) & $>2,000$ & $\mathrm{ND}$ & $\mathrm{ND}$ & $232-1,245(\mathrm{pg} / \mathrm{mL})$ \\
\hline Sodium $(\mathrm{mmol} / \mathrm{L} 9$ & 142 & 145 & 147 & $135-146 \mathrm{mmol} / \mathrm{L}$ \\
\hline Potassium $(\mathrm{mmol} / \mathrm{L})$ & 4.1 & 3.9 & 3.9 & $3.5-5.5 \mathrm{mmol} / \mathrm{L}$ \\
\hline Chloride $(\mathrm{mmol} / \mathrm{L})$ & 108 & 106 & 108 & $98-110 \mathrm{mmol} / \mathrm{L}$ \\
\hline $\mathrm{CO} 2(\mathrm{mmol} / \mathrm{L})$ & 21 & 28 & 25 & $19-34 \mathrm{mmol} / \mathrm{L}$ \\
\hline Anion Gap & 13 & 11 & 14 & 6 to 22 \\
\hline Blood Urea Nitrogen (mg/dL) & 20 & 25 & 30 & $6-20 \mathrm{mg} / \mathrm{dL}$ \\
\hline Osmolality mOsm/Kg & 285 & 291 & 298 & $275-295 \mathrm{mOsm} / \mathrm{kg}$ \\
\hline Creatinine $(\mathrm{mg} / \mathrm{dL})$ & 1.09 & 0.77 & 0.58 & $0.40-1.10 \mathrm{mg} / \mathrm{dL}$ \\
\hline Calcium, Serum (mg/dL) & 7.8 & 7.8 & 7.8 & $8.6-10.3 \mathrm{mg} / \mathrm{dL}$ \\
\hline Protein, Total $(\mathrm{g} / \mathrm{dL})$ & 4.3 & 4.1 & 3.9 & $6.1-8.1 \mathrm{~g} / \mathrm{dL}$ \\
\hline Albumin $(\mathrm{g} / \mathrm{dL})$ & 2.6 & 2.3 & 1.7 & $3.5-5.2 \mathrm{~g} / \mathrm{dL}$ \\
\hline Bilirubin, Total (mg/dL) & 0.3 & 0.5 & 0.5 & $0.0-1.2 \mathrm{mg} / \mathrm{dL}$ \\
\hline AST (SGOT) (U/L) & 66 & 32 & 31 & $10-40 \mathrm{U} / \mathrm{L}$ \\
\hline ALT (SGPT) (U/L) & 70 & 55 & 50 & $0-33 \mathrm{U} / \mathrm{L}$ \\
\hline Alkaline Phosphatase (U/L) & 115 & 122 & 102 & $35-105 \mathrm{U} / \mathrm{L}$ \\
\hline Magnesium (mg/dL) & 1.7 & 1.6 & 1.8 & $1.6-2.6 \mathrm{mg} / \mathrm{dL}$ \\
\hline Prothrombin time (sec) & 20 & NA & NA & $12.0-14.5 \mathrm{sec}$ \\
\hline International Normalized Ratio & 1.65 & NA & 1.1 & NA \\
\hline activated partial thromboplastin time (sec) & 26.1 & NA & NA & $23.7-35.3 \mathrm{sec}$ \\
\hline
\end{tabular}

ALT, alanine aminotransferase; AST, aspartate aminotransferase; NA, not available.

sleep disorders, lethargy, stupor, coma, cerebral edema, and even death [18].

Several mechanisms have been proposed in the developing of GaBHA, which seems to be multifactorial [10]. This syndrome occurs almost exclusively in women, and previous reports have found functional ornithine transcarbamylase (OTC) deficiency given a low enzymatic activity [11, 15], even in the presence of normal OTC sequencing genetic testing $[11,15]$. Others have reported cases of GaBHA with late-onset carbamoyl phosphate synthase 1 (CPS1) deficiency [15]. Nutritional deficits may lead to functional inhibition of urea cycle enzymes, producing accumulation of ammonia [13]. Low arginine levels inhibit the urea cycle [22] as well as low zinc concentrations, possibly by interfering with the OTC function [23]. Therefore, in adult patients who develop GaBHA, OTC deficiency and other urea cycle abnormalities should be strongly considered. RYGB has been associated with hyperinsulinemia and reactive hypoglycemia, which may contribute to the catabolic state and ammonia excess [24]. Also, the gastric-small bowel pouch in RYGB may cause overgrowth of intestinal flora favoring the production of ammonia by urease-producing bacteria [11].

In general, conservative management of hyperammonemic encephalopathy has been traditionally supportive with prevention of seizures and cerebral edema and lactulose and rifaximin [13], achieving reductions in plasma ammonia levels. Other proposed therapies for GaBHA patients have been the repletion of deficient amino acids, zinc, micro nutrients, and intravenous glucose infusion, attempting to improve the catabolic state [10].

\section{Conclusion}

We present the case of a middle-aged woman without prior liver disease, who developed nonfatal hyperammonemic encephalopathy as a late postoperative complication 20 years after RYGB procedure. GaBHA is an underrecognized potentially fatal syndrome, for which early active treatment may be life-saving. It is thought to be associated with hypoalbuminemia, hypoglycemia, and low zinc plasma levels, 


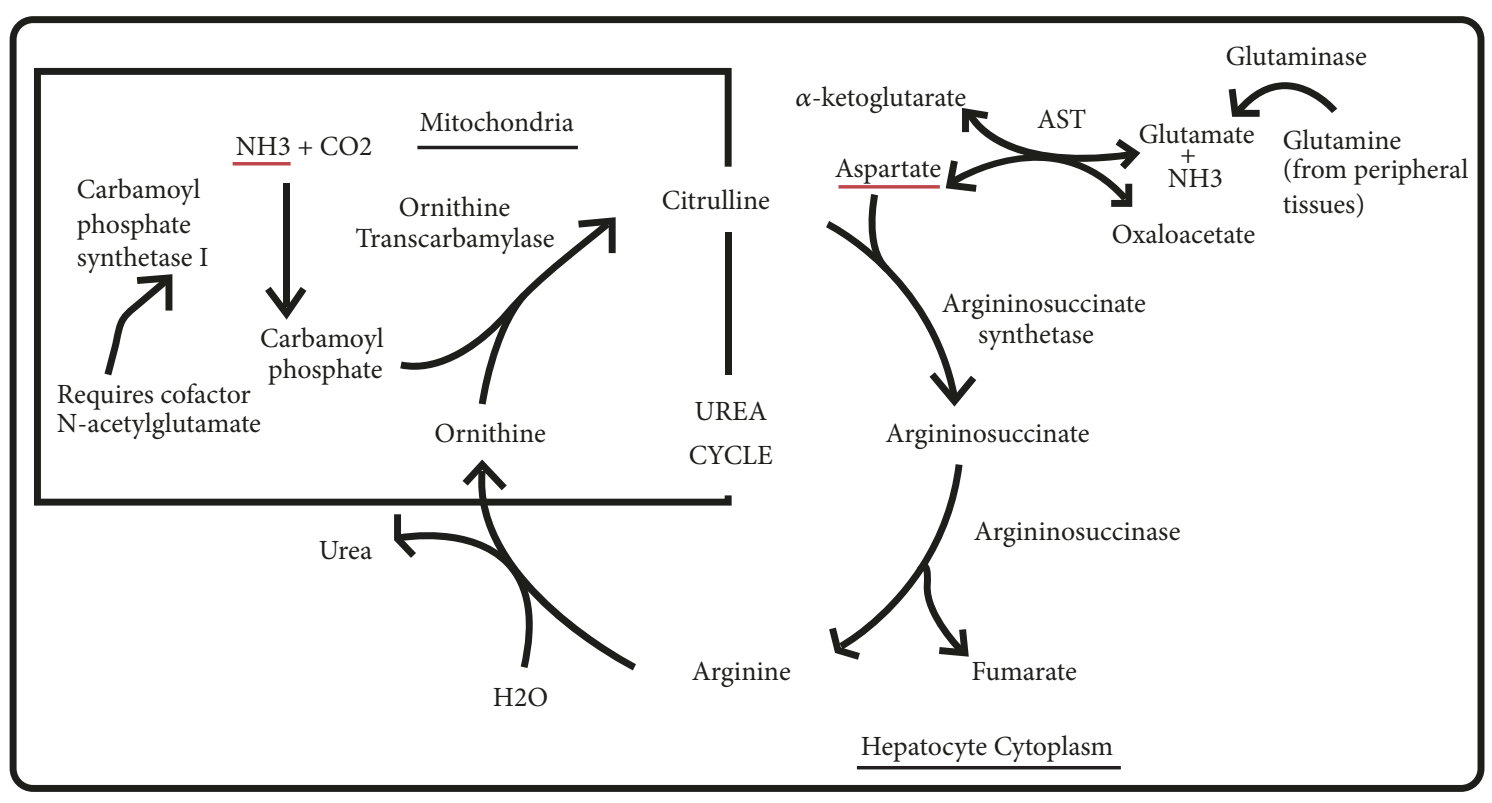

FIGURE 1: Urea cycle. Ammonia is the source of the first amino group in the urea cycle, required in the synthesis of carbamoyl phosphate (CPS), which happens in the hepatocyte mitochondria. The second amino group comes from glutamate in the formation of aspartate, which happens in the hepatocyte cytoplasm. This reaction produces $\alpha$-ketoglutarate, which then becomes available for the Krebs cycle. The synthesis of argininosuccinate links the Krebs cycle and urea cycle. In the peripheral tissues, glutamate accepts free ammonia from amino acid catabolism. Glutamine transports ammonia from the peripheral tissues to the liver, where glutamine is broken back to glutamate and ammonia via glutaminase. AST, aspartate transaminase.

indicating severe nutritional deficiency. In high-risk patients, a rigorous screening for plasma ammonia, zinc, and serum albumin may facilitate an early diagnosis and treatment of this entity.

\section{Conflicts of Interest}

The authors declare that they have no conflicts of interest.

\section{Authors' Contributions}

Author contributions: J.Salcedo and J. Goldstein wrote and edited the manuscript. J. Salcedo and J. Quinonez collected the patient's clinical data. M. Mosetti is the article guarantor.

\section{References}

[1] I. Samuel, E. E. Mason, K. E. Renquist, Y. Huang, M. B. Zimmerman, and M. Jamal, "Bariatric surgery trends: an 18-year report from the International Bariatric Surgery Registry," The American Journal of Surgery, vol. 192, no. 5, pp. 657-662, 2006.

[2] N. T. Nguyen, J. Root, K. Zainabadi et al., "Accelerated growth of bariatric surgery with the introduction of minimally invasive surgery," Archives of Surgery, vol. 140, no. 12, pp. 1198-1202, 2005.

[3] H. Buchwald, Y. Avidor, E. Braunwald et al., "Bariatric surgery: a systematic review and meta-analysis," The Journal of the American Medical Association, vol. 292, no. 14, pp. 1724-1737, 2004.

[4] H. Buchwald and J. N. Buchwald, "Evolution of operative procedures for the management of morbid obesity 1950-2000," Obesity Surgery, vol. 12, no. 5, pp. 705-717, 2002.
[5] D. R. Flum, S. H. Belle, and W. C. King, "Perioperative safety in the longitudinal assessment of bariatric surgery," The New England Journal of Medicine, vol. 361, no. 5, pp. 445-454, 2009.

[6] M. A. Maggard, L. R. Shugarman, M. Suttorp et al., "Metaanalysis: surgical treatment of obesity," Annals of Internal Medicine, vol. 142, no. 7, pp. 547-559, 2005.

[7] K. Seeras and P. P. Lopez, "Roux-en-Y Gastric Bypass, Chronic Complications," in Treasure Island (FL), StatPearls Publishing, 2018, https://www.ncbi.nlm.nih.gov/books/NBK519489/.

[8] B. M. Koffman, L. J. Greenfield, I. I. Ali, and N. A. Pirzada, "Neurologic complications after surgery for obesity," Muscle \& Nerve, vol. 33, no. 2, pp. 166-176, 2006.

[9] K. Juhasz-Pocsine, S. A. Rudnicki, R. L. Archer, and S. I. Harik, "Neurologic complications of gastric bypass surgery for morbid obesity," Neurology, vol. 68, no. 21, pp. 1843-1850, 2007.

[10] A. Nagarur and A. Z. Fenves, "Late presentation of fatal hyperammonemic encephalopathy after roux-En-Y gastric bypass," Baylor University Medical Center Proceedings, vol. 30, no. 1, pp. 41-43, 2017.

[11] A. Z. Fenves, O. A. Shchelochkov, and A. Mehta, "Hyperammonemic syndrome after Roux-en-Y gastric bypass," Obesity, vol. 23, no. 4, pp. 746-749, 2015.

[12] A. Fenves, C. R. Boland, R. Lepe, P. Rivera-Torres, and S. J. Spechler, "Fatal hyperammonemic encephalopathy after gastric bypass surgery," American Journal of Medicine, vol. 121, no. 1, pp. e1-e2, 2008.

[13] J. Estrella, G. Yee, B. Wilcken, M. Tchan, and M. Talbot, "Hyperammonemic encephalopathy complicating bariatric surgery: a case study and review of the literature," Surgery for Obesity and Related Diseases, vol. 10, no. 3, pp. e35-e38, 2014.

[14] M. L. Kromas, O. Y. Mousa, and S. John, "Hyperammonemiainduced encephalopathy: A rare devastating complication of 
bariatric surgery," World Journal of Hepatology, vol. 7, no. 7, p. 1007, 2015.

[15] W. T. Hu, O. H. Kantarci, J. L. Merritt II et al., "Ornithine transcarbamylase deficiency presenting as encephalopathy during adulthood following bariatric surgery," JAMA Neurology, vol. 64, no. 1, pp. 126-128, 2007.

[16] K. L. Borreggine, D. K. Hosker, T. A. Rummans, and D. M. Manning, "Psychiatric manifestations of hyperammonemic encephalopathy following roux-en-y gastric bypass," Psychosomatics, vol. 59, no. 1, pp. 90-94, 2018.

[17] W. L. Stone and G. B. Jaishankar, "Urea cycle disorders," in StatPearls, StatPearls Publishing, 2018, https://www.ncbi.nlm .nih.gov/books/NBK482363/.

[18] W. Barmore and W. L. Stone, "Physiology, urea cycle," in StatPearls, Treasure Island (FL): StatPearls Publishing, 2018, https://www.ncbi.nlm.nih.gov/books/NBK513323/.

[19] R. M. Cohn and K. S. Roth, "Hyperammonemia, bane of the brain," Clinical Pediatrics, vol. 43, no. 8, pp. 683-689, 2004.

[20] D. Xiao, L. Zeng, K. Yao, X. Kong, G. Wu, and Y. Yin, "The glutamine-alpha-ketoglutarate (AKG) metabolism and its nutritional implications," Amino Acids, vol. 48, no. 9, pp. 20672080, 2016.

[21] S. W. Brusilow, "Hyperammonemic encephalopathy," Medicine, vol. 81, no. 3, pp. 240-249, 2002.

[22] C. Bachmann, "Mechanisms of hyperammonemia," Clinical Chemistry and Laboratory Medicine, vol. 40, no. 7, 2002.

[23] E. Aquilio, R. Spagnoli, D. Riggio, and S. Seri, "Effects of zinc on hepatic ornithine transcarbamylase (OTC) activity," Journal of Trace Elements and Electrolytes in Health and Disease, vol. 7, no. 4, pp. 240-241, 1993.

[24] Z. Li, M. L. Yarmush, and C. Chan, "Insulin concentration during preconditioning mediates the regulation of urea synthesis during exposure to amino acid-supplemented plasma," Tissue Engineering Part A, vol. 10, no. 11-12, pp. 1737-1746, 2004. 


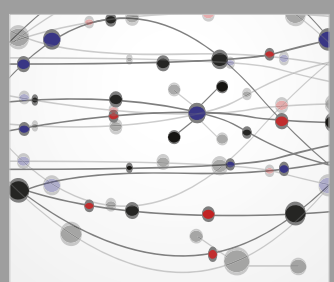

The Scientific World Journal
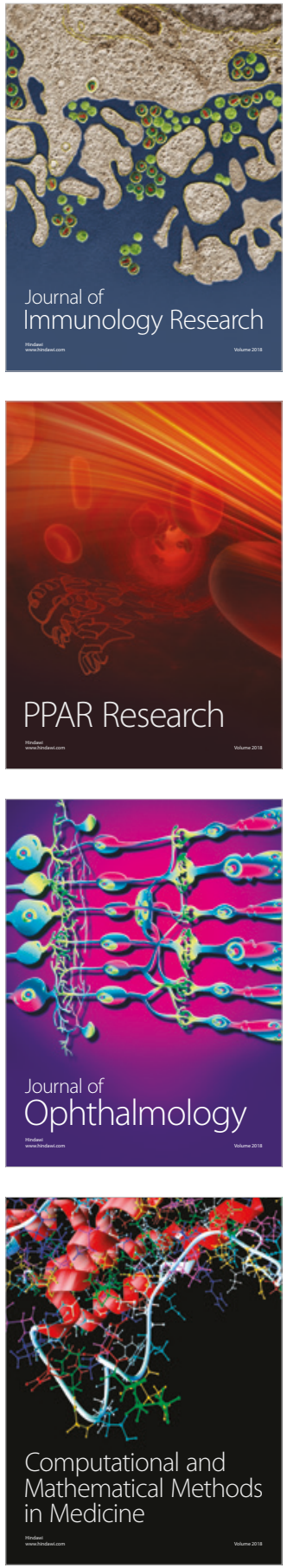

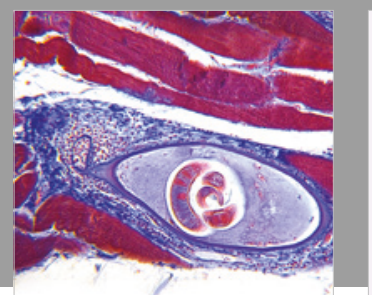

Gastroenterology Research and Practice

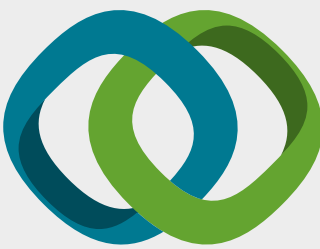

\section{Hindawi}

Submit your manuscripts at

www.hindawi.com
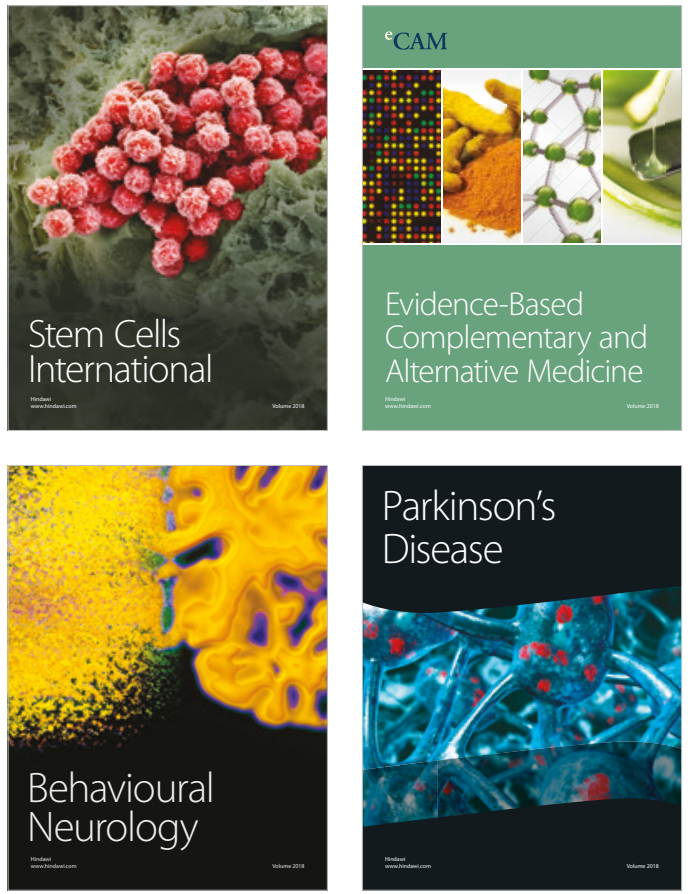

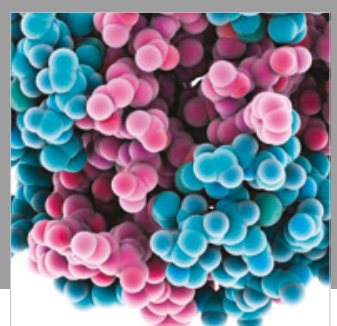

ournal of

Diabetes Research

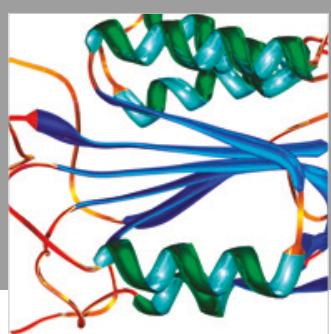

Disease Markers
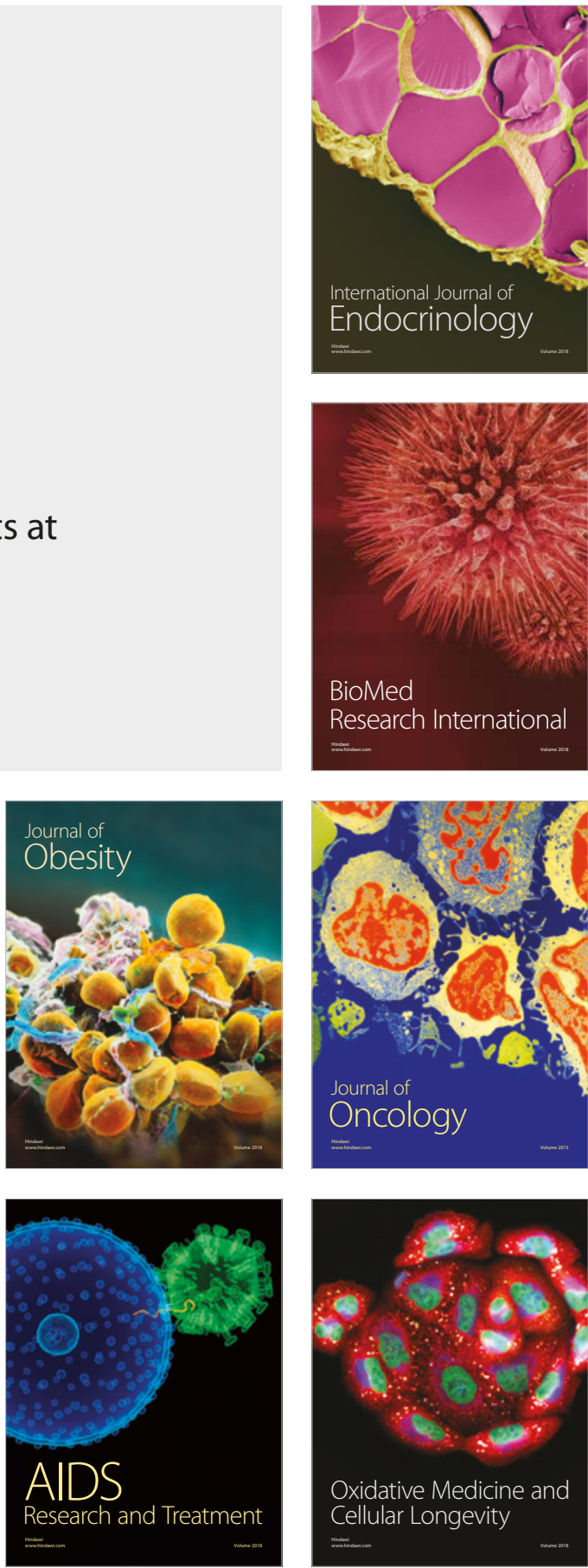